\title{
Investigation of the Extracellular Calcium Effects on Action Potential Using the Most Recent Human Ventricular Cell Models
}

\author{
Chiara Bartolucci ${ }^{1}$, Michelangelo Paci $^{2}$, Stefano Severi ${ }^{1}$ \\ ${ }^{1}$ University of Bologna, Cesena, Italy \\ ${ }^{2}$ Tampere University, Tampere, Finland
}

\begin{abstract}
Which are the quantitative contributions of the mechanisms involved in the relationship between extracellular calcium concentration $\left[\mathrm{Ca}^{2+}\right]_{o}$ and the action potential (AP)? The present work aims to answer this question using human-based modeling and simulations since they could provide useful support to investigate this phenomenon. However, $\left[\mathrm{Ca}^{2+}\right]_{o}$ dependence on AP duration is not reproduced correctly by most of the commonly used human AP models.

Four of the most recent human ventricular AP models have been tested by simulating different extracellular calcium concentrations during the AP-clamp protocol. From earlier studies, it is well known that the L-type $\mathrm{Ca}^{2+}$ current $\left(I_{C a L}\right)$ is the ionic current mainly affected by $\left[\mathrm{Ca}^{2+}\right]_{o}$ changes. In particular, calcium-dependent inactivation (CDI) seems to play the most significant role. For this reason, we simulated two different conditions: with the basal models and with the models in which the CDI has been turned off.

The results pointed out from the models' comparison is that not only $I_{C a L} C D I$ contributes to the APD- $\left[\mathrm{Ca}^{2+}\right]_{o}$ relationship but also the sodium-calcium Exchanger $\left(I_{\text {NaCa }}\right)$ played an important role.
\end{abstract}

\section{Introduction}

Cardiac computational models are convenient tools to investigate the ionic mechanisms involved at the cell level and have already been used in clinical contexts (see, e.g.[1]).

Assessing the dependency of ventricular repolarization on extracellular electrolyte concentrations is essential in all clinical settings where electrolyte changes are involved, e.g., for hemodialysis patients.

It is well-known that extracellular calcium concentration $\left(\left[\mathrm{Ca}^{2+}\right]_{0}\right)$ affects the cardiac action potential (AP): in fact, an increase of $\left[\mathrm{Ca}^{2+}\right]_{0}$ shortens AP while $\left[\mathrm{Ca}^{2+}\right]_{0}$ decrease lengthens it, as observed in different species and cell types [2-4].
L-type $\mathrm{Ca}^{2+}$ current $\left(\mathrm{I}_{\mathrm{CaL}}\right)$ is the ionic current mainly affected by $\left[\mathrm{Ca}^{2+}\right]_{0}$ changes [5], even if there are multiple ionic mechanisms involved in the action potential duration (APD)- $\left[\mathrm{Ca}^{2+}\right]_{0}$ dependence [5]. This phenomenon is not entirely understood yet: the change in $\mathrm{I}_{\mathrm{CaL}}$ driving force resulting from $\left[\mathrm{Ca}^{2+}\right]_{\mathrm{o}}$ increase, alone, should enhance calcium influx; nevertheless, calciumdependent inactivation (CDI) seems to play the major role, thus reducing the current and, consequently, APD.

The use of computational modeling may help to elucidate these complex interactions by analyzing single biophysical mechanisms and their interactions. In this scenario, most of the commonly used human ventricular AP models, which were developed using a single $\left[\mathrm{Ca}^{2+}\right]_{\mathrm{o}}$ value, are not able to reproduce the experimentally observed effects of $\left[\mathrm{Ca}^{2+}\right]_{0}$ changes on APD. $\left[\mathrm{Ca}^{2+}\right]_{0}$ increase prolongs their APDs instead of shortening them, and vice versa (as in the O'Hara-Rudy [6] and the GrandiBers [7] models). Recently, a novel AP ventricular model (Bartolucci et al. [8]) has been published to simulate the correct inverse dependence of APD on $\left[\mathrm{Ca}^{2+}\right]_{\mathrm{o}}$.

In all the models, the $\left[\mathrm{Ca}^{2+}\right]_{0}$ was set to the standard value used in the perfusion bath during voltage-clamp experiments, that is, $1.8 \mathrm{mM}$. This value is quite high compared to the normal serum $\mathrm{Ca}^{2+}$ measured in vivo (1$1.3 \mathrm{mM})$, as discussed in [4]. The variation of $\left[\mathrm{Ca}^{2+}\right]_{0}$ affects the cardiac AP and its duration, and it may lead to arrhythmia onset. For this reason, it is essential to understand the $\left[\mathrm{Ca}^{2+}\right]_{0}$ dependency of repolarization.

In this work, we aim to study and understand the mechanisms underlying the APD- $\left[\mathrm{Ca}^{2+}\right]_{\text {o dependence in }}$ four state-of-the-art human ventricular models (Grandi et al. [7]; O'Hara et al. [6]; Tomek et al. [9]; Bartolucci et al.[8]), which have different behaviors in this particular context.

\section{Methods}

In this work, we considered two well established human ventricular single cell models (Grandi et al. [7]; O'Hara et al. [6]), published in 2010 and 2011, and two 
more recent models (Tomek et al. [9]; Bartolucci et al. [8]), published in 2019 and 2020. Hereafter the models will be referred to as follows: GB, ORd, TorORd, and BPS. Although all these models simulate the human ventricular AP, their different parameters and ionic current formulations produce distinct AP morphologies and properties, e.g., APD or response to the $\left[\mathrm{Ca}^{2+}\right]_{\mathrm{o}}$ variations.

To investigate the effects of $\left[\mathrm{Ca}^{2+}\right]_{0}$ changes on APD and understand the mechanisms behind this relationship, we have designed the following protocol using AP-clamp.

Action potential waveforms (APW) were recorded after steady-state pacing at a constant cycle length $(\mathrm{CL}=1000 \mathrm{~ms})$ in control extracellular solution $\left(\left[\mathrm{Ca}^{2+}\right]_{0}=1.8 \mathrm{mM}\right)$ for each model. The state variables were saved after reaching the steady-state (1000 s) at different $\left[\mathrm{Ca}^{2+}\right]_{\mathrm{o}}(0.9,1.8$, and $2.7 \mathrm{mM})$ and used as initial conditions for the AP-clamp simulations. The same APW was applied as command potential for each $\left[\mathrm{Ca}^{2+}\right]_{0}$, and the currents of interest were studied. We performed the simulations in two conditions: i) with the control model and ii) with a model in which CDI of $\mathrm{I}_{\mathrm{CaL}}$ is turned off.

Model differential equations were implemented in Matlab (Mathworks Inc., Natick, MA, USA) and solved with a variable order solver (ode15s), based on numerical differentiation formulas [10]. $\mathrm{APD}_{\mathrm{X}}$ was measured once membrane voltage reached $\mathrm{X} \%$ of the resting value. The current integrals were obtained using the trapz Matlab function for a duration equal to the corresponding $\mathrm{APD}_{90}$ values.

\section{Results}

In Figure 1, the behavior of the models in terms of AP and $\mathrm{I}_{\mathrm{CaL}}$ can be observed for three different values of $\left[\mathrm{Ca}^{2+}\right]_{0}$ in steady-state. As shown, $\left[\mathrm{Ca}^{2+}\right]_{0}$ markedly affects the AP morphology and duration, and $\mathrm{I}_{\mathrm{CaL}}$ time courses look different in the four models. The dependence between APD and $\left[\mathrm{Ca}^{2+}\right]_{0}$ is reported in Figure 2. BPS is the only model reproducing the inverse relationship. Conversely, both ORd and TorORd reproduce a nonphysiological direct dependence, which is further exacerbated in the GB model.

To understand the mechanisms underlying this behavior, we quantified the contribution of CDI, by evaluating the $\mathrm{I}_{\mathrm{CaL}}$ integral during the AP-clamp protocol. The currents were obtained with the four control models (VDI+CDI) and with the same models where CDI was turned off (VDI only).
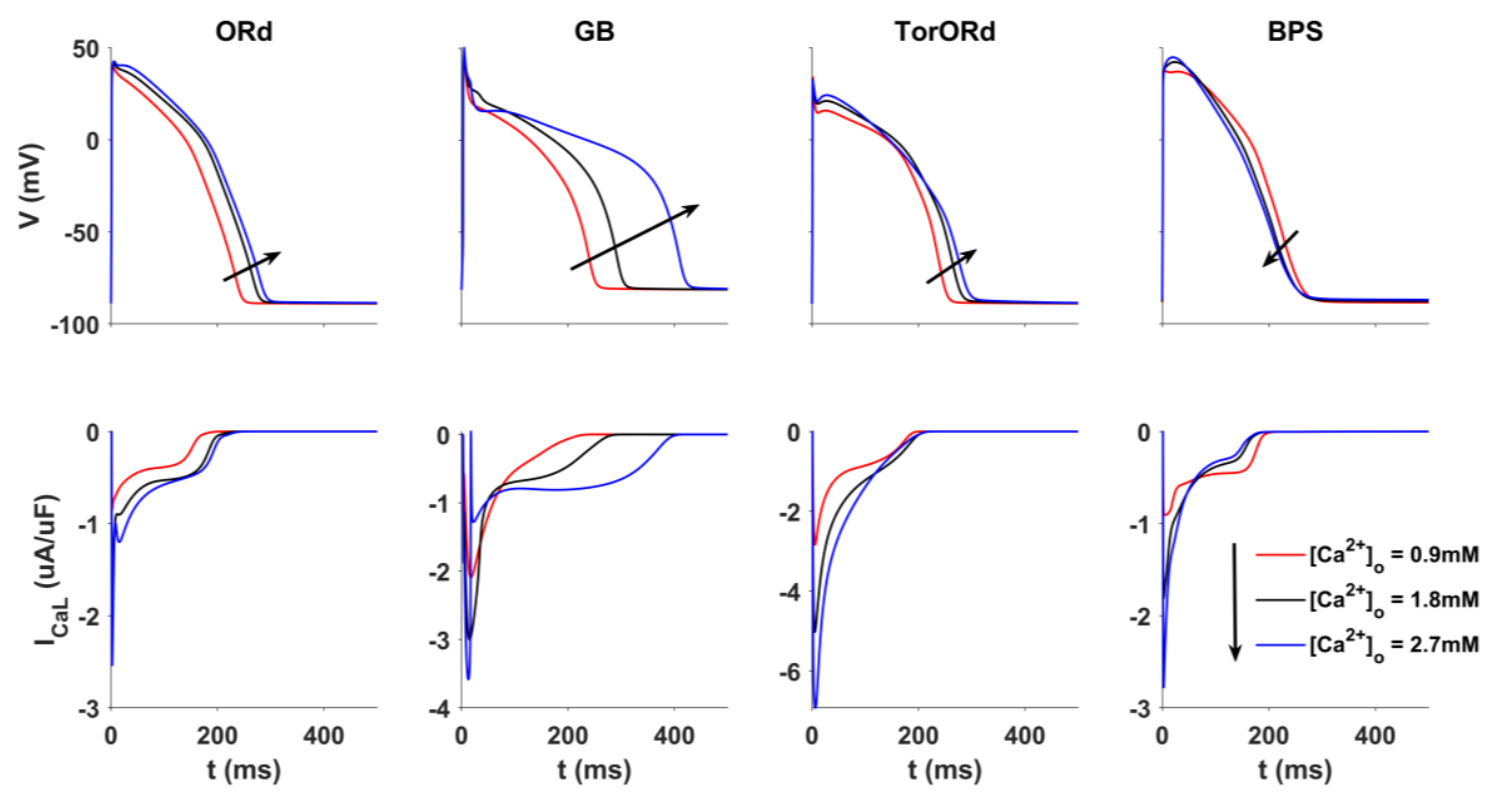

Figure 1. Comparison between the four models (ORd, GB, TorORd, BPS): action potentials (top) and $\mathrm{I}_{\mathrm{CaL}}$ traces (bottom), at three different $\left[\mathrm{Ca}^{2+}\right]_{\mathrm{o}}$ values $(0.9 \mathrm{mM}-$ red; $1.8 \mathrm{mM}-$ black; $2.7 \mathrm{mM}-$ blue $), \mathrm{CL}=1 \mathrm{~s}$ in steady state. 


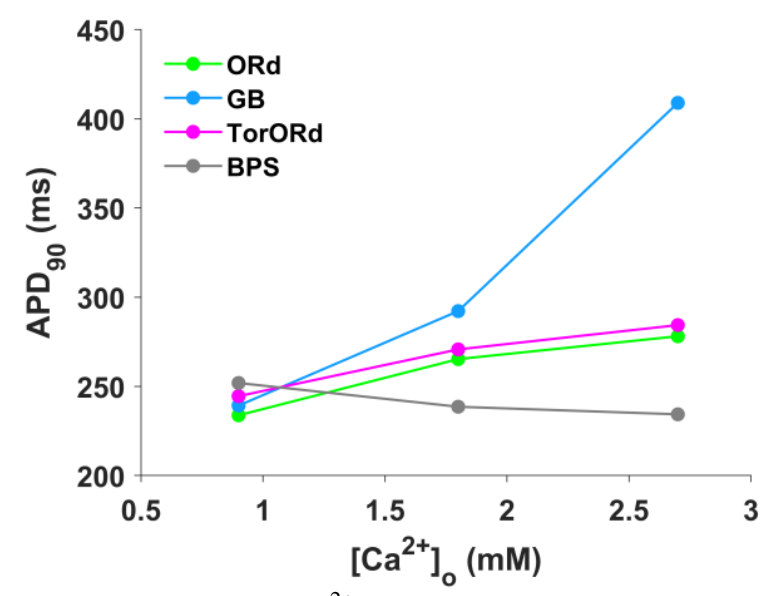

Figure 2. APD- $\left[\mathrm{Ca}^{2+}\right]_{0}$ relationship for $\mathrm{ORd}, \mathrm{GB}$, TorORd, and BPS models in the range $0.9-2.7 \mathrm{mM}$.
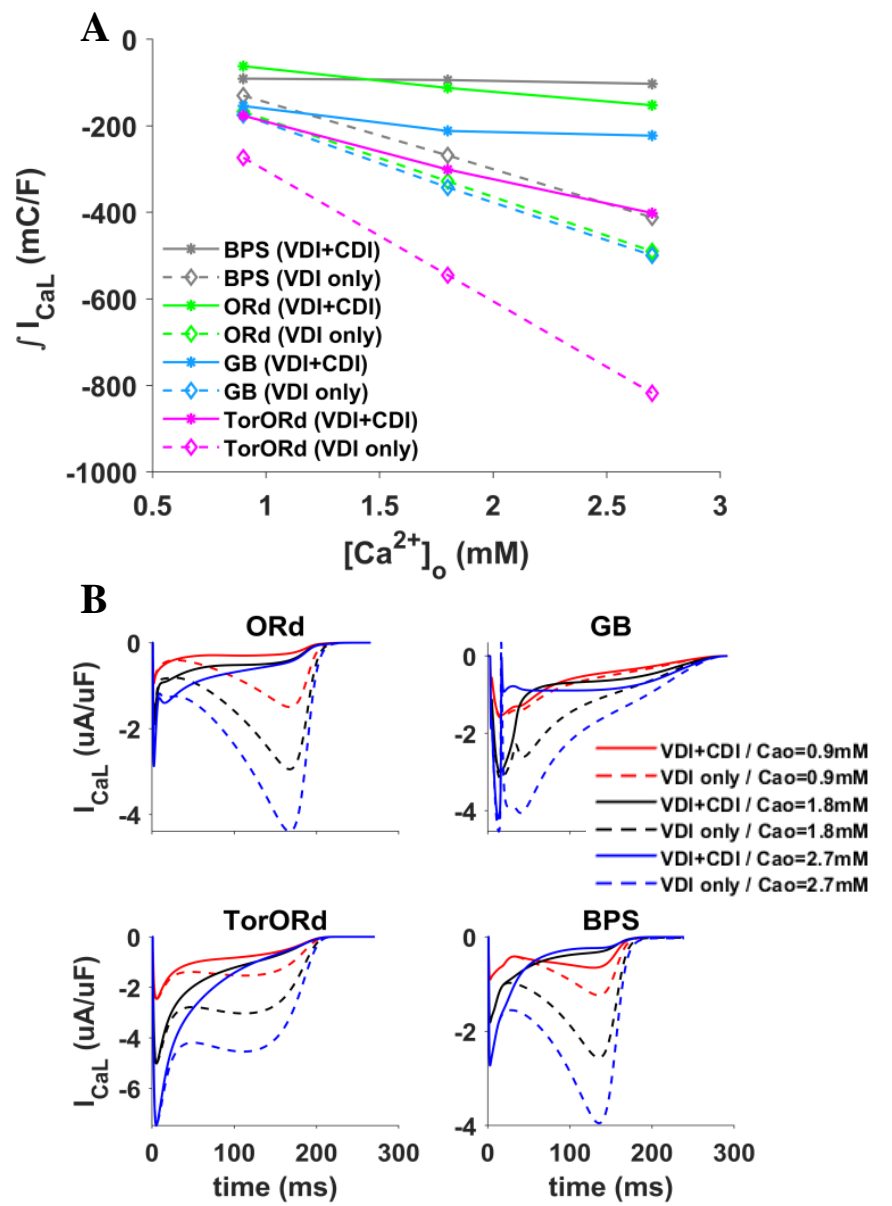

Figure 3. (A) Integral of $I_{C a L}$ currents and (B) $I_{C a L}$ traces for ORd, GB, TorORd, and BPS models in the range $0.9-2.7 \mathrm{mM}$ with the control (VDI+CDI) and VDIonly evaluated in AP-clamp.

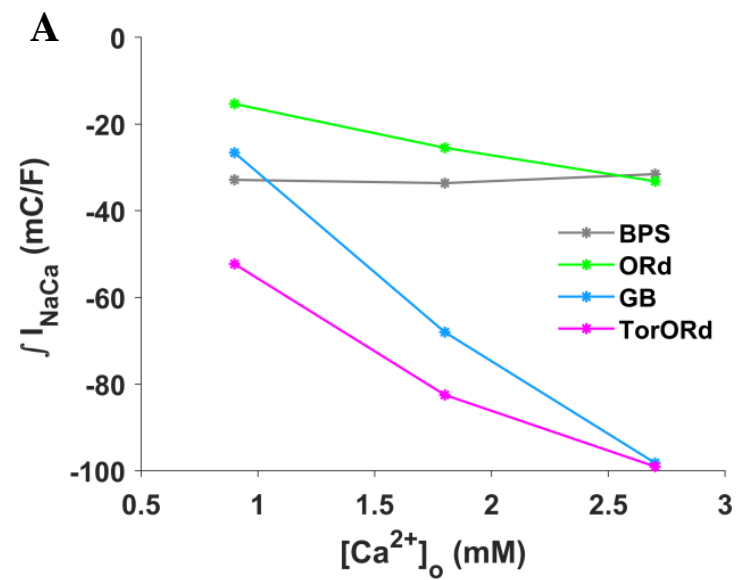

B
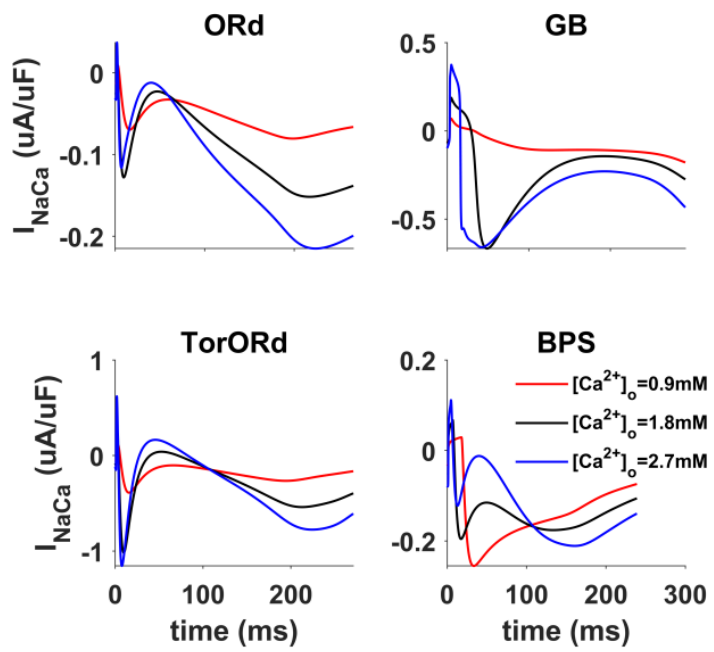

Figure 4. (A) Integral of $\mathrm{I}_{\mathrm{NaCa}}$ currents and (B) $\mathrm{I}_{\mathrm{NaCa}}$ traces for ORd, GB, TorORd, and BPS models in the range $0.9-2.7 \mathrm{mM}$ during AP-clamp.

Figures 3A-B show the integral of $\mathrm{I}_{\mathrm{CaL}}$ currents $\left(\int \mathrm{I}_{\mathrm{CaL}}\right)$ and the $\mathrm{I}_{\mathrm{CaL}}$ traces for the four models at different $\left[\mathrm{Ca}^{2+}\right]_{\mathrm{o}}$ in VDI+CDI conditions (solid lines) and VDI-only (dashed lines). In all the models turning off the CDI leads to a strong linear dependence of $\int \mathrm{I}_{\mathrm{CaL}}$ on $-\left[\mathrm{Ca}^{2+}\right]_{\mathrm{o}}$. In the BPS model, this dependence is almost completely lost when the CDI is present: this highlights the strong CDI effect on $\mathrm{I}_{\mathrm{CaL}}$ in BPS. Since this model correctly reproduces the APD- $\left[\mathrm{Ca}^{2+}\right]_{\mathrm{o}}$ dependence, CDI might be its primary cause, as indeed described in [8]. Conversely, CDI impacts less $\mathrm{I}_{\mathrm{CaL}}$ in TorORd and ORd, as shown by the small slope change in Figure 3A. The GB model shows a slope change similar to BPS (in particular for $\left[\mathrm{Ca}^{2+}\right]_{0}=1.8$ and $\left.2.7 \mathrm{mM}\right)$, but the APD- $\left[\mathrm{Ca}^{2+}\right]_{\mathrm{o}}$ dependence is not physiological (Figure 2, cyan trace). For this reason, with the same approach, we analyzed the other currents affected by $\left[\mathrm{Ca}^{2+}\right]_{\mathrm{o}}$ variations. This investigation pointed out the contribution of $\mathrm{I}_{\mathrm{NaCa}}$. As can be seen in Figures $4 \mathrm{~A}-\mathrm{B}$, the integral of $\mathrm{I}_{\mathrm{NaCa}}$ remains almost 
constant at different $\left[\mathrm{Ca}^{2+}\right]_{0}$ values in BPS. Instead, the GB $\mathrm{I}_{\mathrm{NaCa}}$ has a significant dependence on $\left[\mathrm{Ca}^{2+}\right]_{0}$ : if $\left[\mathrm{Ca}^{2+}\right]_{\mathrm{o}}$ increases, the inward $\mathrm{I}_{\mathrm{NaCa}}$ indeed increases, and the APD will prolong as a consequence.

\section{Discussion and Conclusions}

It is well established that a change in $\left[\mathrm{Ca}^{2+}\right]_{0}$ may affect many electrogenic transport mechanisms across the membrane. The result of our analysis was that three ventricular models (ORd, GB, and TorORd) responded with APD prolongation to $\left[\mathrm{Ca}^{2+}\right]_{0}$ increase (Figure 2). This behavior is in contrast to the APD shortening observed in vitro and in vivo when extracellular, or plasma calcium concentration, is increased. The BPS model correctly reproduced this dependence.

A possible explanation for the observed discrepancy consists of underestimating the $\left[\mathrm{Ca}^{2+}\right]$ sensitivity of $\mathrm{I}_{\mathrm{CaL}}$ CDI in the ORd, GB, and TorORd models. As a consequence, upon an increase in $\left[\mathrm{Ca}^{2+}\right]_{\mathrm{o}}$, the stronger driving force overcame CDI-induced $\mathrm{I}_{\mathrm{CaL}}$ reduction. Thus, the outcome was a net current increase during AP phase 2 and consequent APD prolongation. To prove this point, we recently developed the BPS model [8] starting from ORd and directly manipulating CDI: indeed, the strong $\left[\mathrm{Ca}^{2+}\right]$ sensitivity of CDI, as in the BPS model, enables simulating APD prolongation at decreasing $\left[\mathrm{Ca}^{2+}\right]_{0}$. Overall, these results support the hypothesis of CDI as the mechanism primarily responsible for the APD- $\left[\mathrm{Ca}^{2+}\right]_{0}$ dependency.

In the GB model, this behavior is less evident (i.e., in phase 2, smaller $\mathrm{I}_{\mathrm{CaL}}$ increment can be appreciated during $\left[\mathrm{Ca}^{2+}\right]_{\mathrm{o}}$ variations), but still, the APD- $\left[\mathrm{Ca}^{2+}\right]_{\mathrm{o}}$ dependence was simulated nonphysiologically. Besides $\mathrm{I}_{\mathrm{CaL}}, \mathrm{I}_{\mathrm{NaCa}}$ could also play a role. Simulations show quite different patterns of $\mathrm{I}_{\mathrm{NaCa}}$ during the AP plateau (Figure 4B). In particular, for $\mathrm{GB}, \mathrm{I}_{\mathrm{NaCa}}$ integral strongly depends on the $\left[\mathrm{Ca}^{2+}\right]_{\mathrm{o}}$ variations (Figure $4 \mathrm{~A}$ ), very likely giving a key contribution to the APD observed prolongation.

The use of the AP-clamp protocol allowed us to analyze the effect of $\left[\mathrm{Ca}^{2+}\right]_{0}$ variations on each membrane current, independently of closed-loop effects of membrane potential changes. A deeper analysis might focus on possible effects on APD from altered $\left[\mathrm{Ca}^{2+}\right]_{\mathrm{i}}$; however, also such effects on APD would be mediated by $\left[\mathrm{Ca}^{2+}\right]_{i}$ sensitive membrane currents, i.e. $\mathrm{I}_{\mathrm{CaL}}, \mathrm{I}_{\mathrm{NaCa}}$. A limitation of this study is the lack of specific experimental validation, for which specifically designed measurements on human ventricular cardiomyocytes are needed, which are not currently available in the literature.

In this scenario, the BPS model expands the current domain of applicability of computational models of human cardiac ventricular AP. It is indeed suitable to explore changes in ventricular electrophysiology induced by electrolyte variations (e.g., effects of hemodialysis or pathological changes in $\mathrm{Ca}^{2+}$ concentrations). In conclusion, this study tests potential mechanisms underlying the inverse APD- $\left[\mathrm{Ca}^{2+}\right]_{0}$ dependence; these results support the role of CDI as the primary mechanism. The actual function of $\mathrm{I}_{\mathrm{NaCa}}$ in modulating APD is far from being fully understood and deserves a more detailed analysis based on a thorough review of the available experimental data.

\section{References}

[1] Severi S, Rodriguez B, Zaza A. Computational cardiac electrophysiology is ready for prime time. Europace 2014;16:382-3. doi:10.1093/europace/euu044.

[2] Leitch S. Effect of Raised Extracellular Calcium on Characteristics of the Guinea-pig Ventricular Action Potential. J Mol Cell Cardiol 1996;28:541-51. doi:10.1006/jmcc.1996.0050.

[3] Kass RS. Control of action potential duration by calcium ions in cardiac Purkinje fibers. J Gen Physiol 1976;67:599-617. doi:10.1085/jgp.67.5.599.

[4] Severi S, Corsi C, Cerbai E. From in vivo plasma composition to in vitro cardiac electrophysiology and in silico virtual heart: the extracellular calcium enigma. Philos Trans A Math Phys Eng Sci 2009;367:2203-23.

[5] Grandi E, Pasqualini FS, Pes C, Corsi C, Zaza A, Severi S. Theoretical investigation of action potential duration dependence on extracellular $\mathrm{Ca} 2+$ in human cardiomyocytes. J Mol Cell Cardiol 2009;46:332-42.

[6] O'Hara T, Virág L, Varró A, Rudy Y. Simulation of the undiseased human cardiac ventricular action potential: Model formulation and experimental validation. PLoS Comput Biol 2011;7.

[7] Grandi E, Pasqualini FS, Bers DM. A novel computational model of the human ventricular action potential and $\mathrm{Ca}$ transient. $\mathrm{J}$ Mol Cell Cardiol 2010;48:112-21.

[8] Bartolucci C, Passini E, Hyttinen J, Paci M, Roth BJ. Simulation of the Effects of Extracellular Calcium Changes Leads to a Novel Computational Model of Human Ventricular Action Potential With a Revised Calcium Handling 2020;11:1-20. doi:10.3389/fphys.2020.00314.

[9] Tomek J, Bueno-Orovio A, Passini E, Zhou X, Minchole A, Britton O, et al. Development, calibration, and validation of a novel human ventricular myocyte model in health, disease, and drug block. Elife 2019;8. doi:10.7554/eLife.48890.

[10] Shampine LF, Reichelt MW. The MATLAB ODE Suite. SIAM J Sci Comput 1997;18:1-22.

Address for correspondence:

Stefano Severi

Department of Electrical, Electronic

and Information Engineering,

University of Bologna,

Via dell'Università 50, 47522 Cesena (FC),

Italy

stefano.severi@unibo.it 